\title{
Perioperative Management of Neonates Born to Mothers with COVID-19 Undergoing Urgent Cardiac Surgery
}

\author{
Nabil A Mageed* and Ibrahim I Abd El Baser \\ Department of Anesthesia and Surgical Intensive Care, Faculty of Medicine, Mansoura University, Egypt
}

*Corresponding author: Nabil A Mageed, Department of Anesthesia and Surgical Intensive Care, Mansoura University, Egypt.

\author{
Received Date: April 19, 2020 \\ Published Date: April 27, 2020
}

\begin{abstract}
Neonates born to mothers with COVID-2019 are at high risk of SARS-CoV-2 infection, although the direct vertical transmission from mother to fetus have not been documented but the baby may be infected during breast feeding by airborne or droplet infection. The baby should be isolated from infected mother and from other neonates born to healthy mothers. Neonates born with critical and severe cardiac anomalies usually require lifesaving cardiac surgical intervention. The patient's hemodynamics should be stabilized before surgery. The patient should be considered as a source of SARS-CoV-2 transmission of infection even in the absence of any clinical, laboratory or radiologic evidence of COVID-2019. Medical personnel's should use personal protective equipment (PPE) especially during intubation, extubation and handling patient secretions. The circuits of cardiopulmonary bypass should be small as possible to decrease the priming volume. This review is focused on anesthetic hemodynamic goals of different congenital heart disease and methods of protection of medical team in operating room and neonatal intensive care unit from transmission of SARS-CoV-2 infection.
\end{abstract}

Keywords: Neonates; mothers; COVID-19; Cardiac; Surgery

\section{Introduction}

In December 2019 outbreaks of fever and atypical viral bronchopneumonia have occurred in Wuhan, China [1]. The causative viral organism of this atypical bilateral bronchopneumonia was known as severe acute respiratory syndrome coronavirus 2 (SARS-CoV-2) and the disease have been named COVID-2019. It can be transmitted from human to the other causing severe pneumonia and death [2]. It is a highly aggressive virus that have been transmitted to almost all countries in the world causing life threatening pneumonia. Pregnant women are at higher risk of SARSCoV-2 infection due to their depressed immunity that makes them and their fetuses at direct threat [3]. Neonates have been proven to be at high risk of SARS-CoV-2 infection when they are delivered by infected mothers [4], although the direct vertical intrauterine transmission from mother to fetus have not been documented [5].

In living neonates, about $0.25-0.3 \%$ of live birth have critical congenital heart disease (CHD) and it is the main cause of morbidity and mortality in neonates even with the antenatal diagnosis [6]. Some forms of severe CHD in the neonatal period require immediate live saving intervention either by surgery or cardiac catheterization [7]. The severe forms of CHD which require early intervention in the first days of neonatal life include obstructed total anomalous pulmonary venous connection (TAPVC), hypoplastic left heart syndrome (HLHS) with intact atrial septum, critical aortic stenosis, pulmonary atresia, tricuspid atresia, severe coarctation of the aorta with closed ductus arteriousus and d-transposition of the great arteries (DTGA).

In this review, we discuss methods of transmission and pathogenesis of SARS-CoV-2 infection in neonates born to infected mothers, the forms and hemodynamic anesthetic goals of severe CHD that require surgical or trans-catheter intervention in the first days of neonatal life and how to protect the medical personnel's in operating room and neonatal intensive care unit (NICU) from catching SARS-CoV-2 infection. 


\section{SARS-CoV-2 Infection}

Outbreaks of SARS-CoV-2 is still occurring and spreading all over the world. Pregnant women are more susceptible to infection but the increased risk of perinatal complications such as abortion, intrauterine fetal death, preterm labor, fetal dysrhythmia and fetal respiratory distress is not documented. In a study from Iran, three infected women with SARS-CoV-2 delivered three neonates, two mothers of these 3 infected cases developed acute respiratory distress syndrome (ARDS) following labor and died while their babies were negative when they tested for SARS-CoV-2 infection [8]. SARS-CoV-2 infection in pregnant women may increase the risk of parturient morbidity and mortality. Chen $\mathrm{R}$, et al. [5] reviewed the recorded clinical data, high resolution chest computed tomography (CT) and laboratory investigation in 9 pregnant ladies with confirmed SARS-CoV-2 infection who were referred to Zhongnan Hospital of Wuhan University. They found that, the severity of clinical manifestations was similar with that of non-pregnant SARSCoV-2 infected women. All 9 infected mothers delivered 9 living neonates and no parturient developed severe SARS-CoV-2 or died, and all born neonates were negative when tested virus.

\section{Congenital and Postnatal SARS-CoV-2 Transmission}

The data of vertical transmission of SARS-CoV-2 from infected pregnant women to their fetuses are limited and the risk of severe respiratory complications and mortality is higher than nonpregnant infected woman. Neonates can catch SARS-CoV-2 infection from infected mothers during breast feeding through respiratory droplets, therefore, infected mothers should wear face mask and considers contact standard precautions during breastfeeding [9].

Two studies reported the presence of SARS- CoV- 2 - specific IgM and IgG in 3 neonates born to infected mothers, giving the possibility of trans placental transfer to the fetus with subsequent fetal production of IgM antibody [10,11]. But, in other diseases false- positive IgM tests have been described, thus making the interpretation of these two studies doubtful, so further studies will be needed to assess the potential risk of vertical transmission [12].

\section{Care for Neonates Born to Infected Mothers with COVID-19}

The experience of neonatal care for babies born to infected mothers with SARS-CoV-2 is limited. Zhu H, et al. [13], reported different clinical manifestations in neonates born to mothers with COVID-19, some neonates developed dyspnea (6 newborn) , cyanosis in three newborn, bleeding from gastrointestinal tract in two babies, multiple organ dysfunction and disseminated coagulopathy in one baby. Despite of these clinical presentations, nasopharyngeal swabs testing for SARS-CoV-2 were negative in all neonates. The above findings indicate that these neonatal complications may not be attributed to in utero transmission. It is hypothesized that neonates born to infected mothers with SARSCoV-2 may become infected during delivery or in the uterus. For this reason, the baby must be isolated from other neonates to avoid the possibility of transmission of SARS-CoV-2 infection. It is wise to keep healthy babies in the room of non-infected mother, but infected mother should be temporary separated from her baby as in the case the epidemic of the H1N1 [14]. Skin-to-skin contact should be avoided between the neonate and mother at risk of infection. Mother with suspected SARS-CoV-2 infection should be temporarily separated from their newborn baby and kept in another separate room. High risk neonates (preterm, presence of respiratory distress and immune compromised baby) should be absolutely separated from mothers who are susceptible or with confirmed SARS-CoV-2 infection.

\section{Critical CHD in Neonates}

\section{Duct flow dependent CHD}

In this form of CHD, the normal blood flow from the left ventricle to descending aorta may be compromised. Patency of ductus arteriosus bypass the obstructing cardiac defect and maintains the distal systemic circulation. Physiologic closure of the duct deprives the distal descending aorta from the shunted systemic blood flow resulting in tissue hypoperfusion, hemodynamic instability, and lactic acidosis [15].

Duct flow dependent CHD can be classified as follow:

a) To maintain distal blood flow in descending aorta in case of left sided obstructive lesions. This occurs in case of hypoplastic left heart syndrome, critical aortic stenosis and severe coarctation of aorta with or without interrupted aortic arch.

b) To maintain blood flow in pulmonary circulation in case of right sided intra-cardiac obstructive lesions. These lesions include single ventricle with pulmonary atresia, severe pulmonary stenosis or atresia with intact inter-ventricular septum, simultaneous pulmonary and tricuspid atresia and severe Ebstein's anomaly.

c) To ensure adequate mixing of two separate circulation as in transposition of great arteries (TGA).

\section{Foramen ovale flow dependent CHD}

Wide communication between the two atria is lifesaving in newborn to maintain hemodynamic stability. Patent foramen ovale relieve lung congestion in case of mitral atresia and hypoplastic left heart syndrome. It prevents excessive pressure load on the right side of the heart and maintains the compromised systemic blood flow in case of total anomalous pulmonary venous connection (TAPVC), tricuspid atresia and pulmonary atresia with intact septum.

\section{Preoperative Preparation}

Neonates with critical CHD born to mothers with susceptible or confirmed SARS-CoV-2 infection require specific perioperative care to stabilize the neonatal hemodynamics and to protect the medical personnel's from transmission of SARS-CoV-2 infection. The newborn of infected mothers should be considered as a source of 
transmission of SARS-CoV-2 infection, even neonates with negative throat swab. The patients should be isolated from well-known infected mothers.

Preoperative clinical assessment and perinatal history would help to know cardio-respiratory functional status. Reviewing the reports of echocardiography and cardiac catheter help to choose the appropriate anesthetic management plan. Complete blood count, coagulation and bleeding studies, hepatic and renal function tests should be reviewed. Preoperative hematocrit more than $60 \%$ requires perioperative hemodilution to decrease blood viscosity, thus avoiding thromboembolic complications and improving tissue perfusion. Analysis of serum lactate and arterial blood gas (ABG) can help to assess the adequacy of systemic and pulmonary perfusion. Clear fluids containing glucose are allowed up to 2 to 3 hours and milk up to 4 hours before induction of anesthesia [16]. Premedication is restricted to atropine $10 \mu \mathrm{g} / \mathrm{kg}$ or glycopyrrolate $5 \mu \mathrm{g} / \mathrm{kg}$ given intramuscular 15 minute before surgery [17]. Neonates and infants with hemodynamic instability or cardiogenic shock may benefit from prostaglandin E1 infusion that can be administered via peripheral cannula (or interosseous line) to maintain ductus arteriosus patency and should be continued till initiation of cardiopulmonary bypass (CPB) is initiated [18]. In neonates with severe pulmonary hypertension, the perioperative anesthetic goal is directed to avoid pulmonary hypertensive crisis which is associated increased neonatal mortality [19].

\section{Intra-operative Management}

\section{Operating room measures to protect medical personnel's}

The aim of these measure is to minimize exposure to patient's secretions. Hand hygiene by frequent washing of both hands with alcohol-based gels particularly after gloves removal, before handling anesthesia machine, after contact with contaminated subjects and after any contact with the patient. Operating room personnel's should use personal protective equipment (PPE) as shown in figure 1. A well-fitting N95 masks can protect against airborne and droplet transmission of infection with SARS-CoV-2 but powered air-purifying respirator (PAPR) is superior to N95 masks. During intubation and extubation anesthetists must wear protective gloves, N95 masks/ PAPR, gown and eye shield with the use of disposable sterile equipment for neonatal intubation. The use of prophylactic antiemetic can reduce the incidence of vomiting and possibility of spread of SARS-CoV-2 infection (Figure 1).

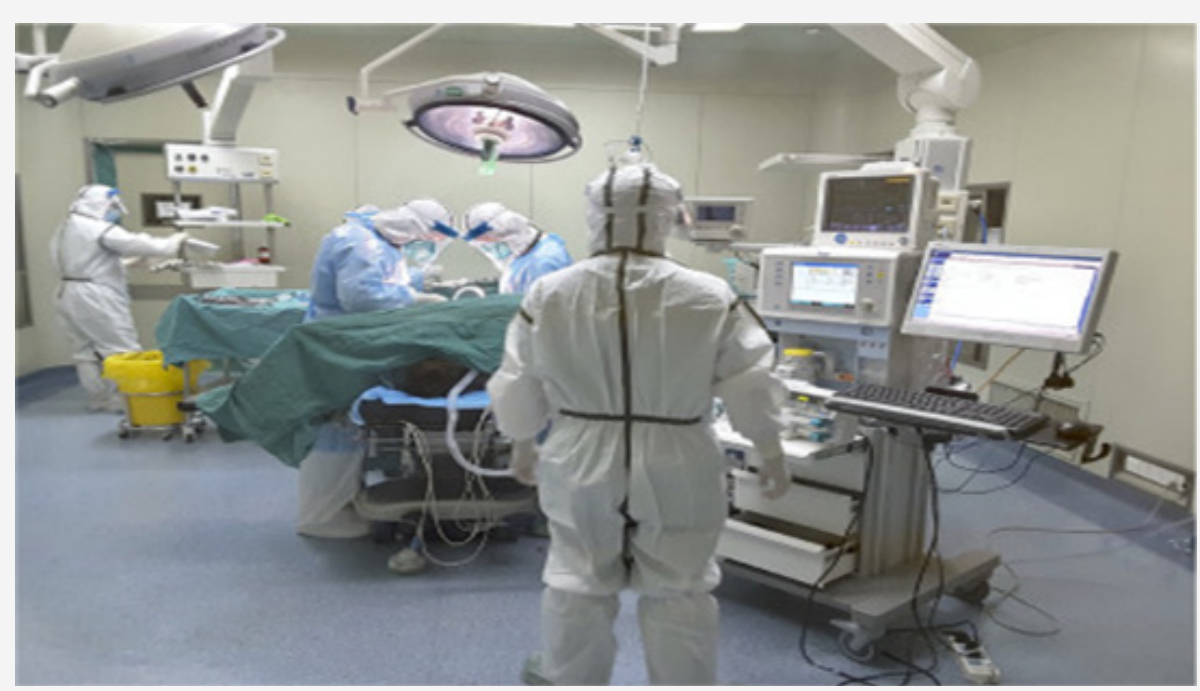

Figure 1: Personal protective equipment (PPE) during emergency surgery [20].

\section{Monitoring}

The basic monitoring of neonates undergoing cardiac surgery includes, pulse oximetry, capnography, 5-leads electrocardiography, invasive arterial blood pressure monitoring and central venous pressure monitoring. Some cardiac lesions require some modifications for patient monitoring. Duct dependent critical cardiac lesions require pulse oximetry monitoring of both upper and lower limbs due the discrepancy of arterial oxygen saturation between the upper and lower half of the body. Invasive arterial pressure monitoring from the right arm is essential in coarctation of aorta and interrupted aortic arch surgeries. In patients who require Glenn bidirectional shunt the central venous catheter should be placed in femoral vein.

\section{Induction and maintenance of anesthesia}

In neonates with difficult venous access, sevoflurane is frequently used to induce anesthesia. Ketamine is the ideal intravenous agent for induction of anesthesia in patients with cyanotic heart disease and patients with pulmonary hypertension as it doesn't decrease systemic vascular resistance and increases mean arterial pressure [21,22]. Etomidate maintains the patient hemodynamics but may cause adrenal insufficiency. Propofol is contraindicated in neonates and it markedly decreases systemic vascular resistance and systemic arterial pressure, which affect shunt dynamics [21,22]. Pancuronium is the muscle relaxant of choice in neonates as it causes tachycardia which counteract the bradycardic effect of the high doses of opioid. The combined use of 
pancuronium and fentanyl is associated with stable hemodynamics. Neonates are intubated with 3 to $3.5 \mathrm{~mm}$ endotracheal tube either by oral or nasal route. Nasal intubation is more comfortable to the newborn patient and more stable with low incidence of inadvertent extubation. Central venous and arterial catheters are secured, and a urinary catheter of suitable size is inserted. The anesthetic hemodynamic goals of CHD are summarized in figures (2\&3). Anesthesia can be maintained with sevoflurane or isoflurane as both inhalational don't affect the shunt fraction [23]. Prophylactic antibiotics are administered before skin incision according to the guidelines of each surgical center. Neonates are at high risk of bleeding and will benefit from administration of antifibrinolytic drugs such as tranexamic acid. Administration of steroids (methylprednisolone) may reduce the risk of systemic inflammatory response to cardiopulmonary bypass (Figure $2 \& 3$ ).

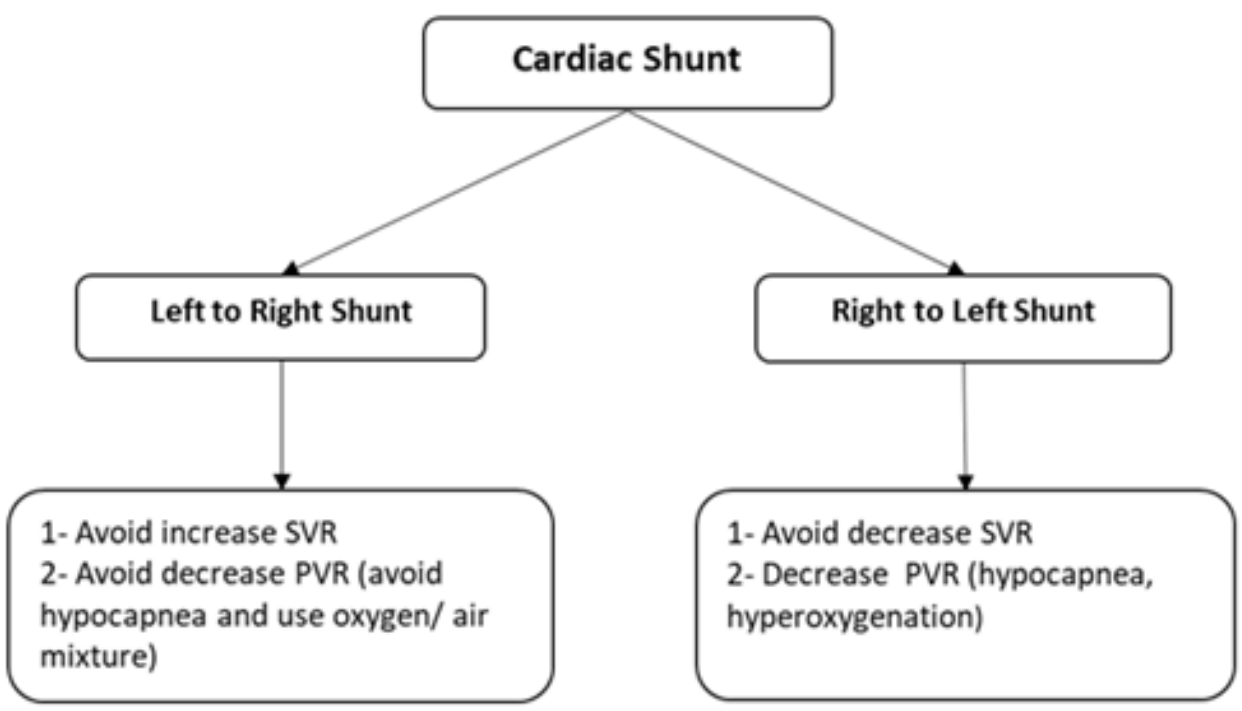

Figure 2: Anesthetic hemodynamic goals of congenital cardiac shunts. SVR: systemic vascular resistance, PVR: pulmonary vascular resistance.

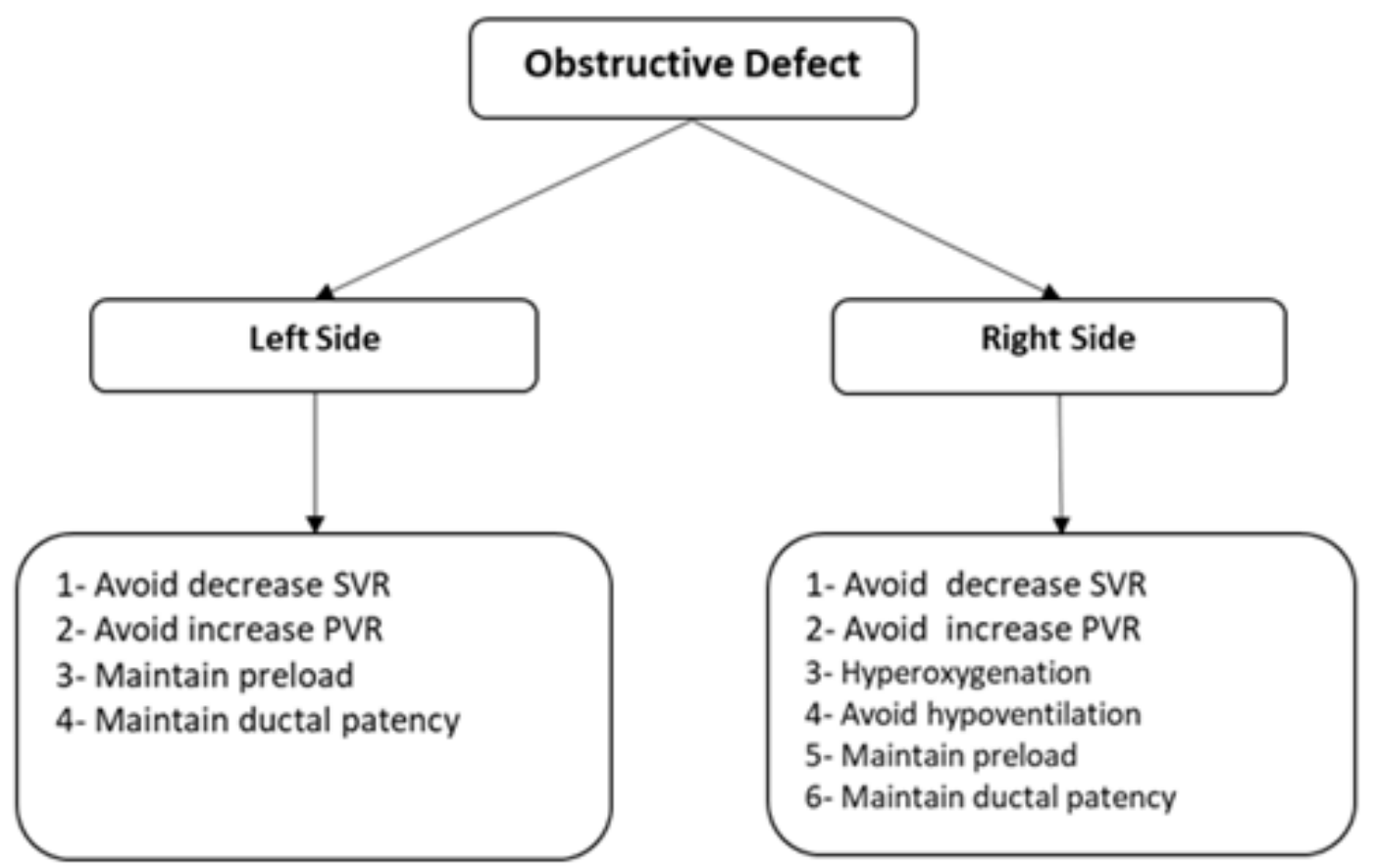

Figure 3: Anesthetic hemodynamic goals of obstructive cardiac lesions. SVR: systemic vascular resistance, PVR: pulmonary vascular resistance. 


\section{Management of CPB}

Heparin is administered in a dose of 3-4 mg via a central vein to increase the activated clotting time (ACT) more than 480 seconds before initiation CPB. The primary aim of improving the outcome of CPB in neonates is to minimize the contact area between $\mathrm{CPB}$ circuits and blood by making more smaller circuits [24]. The neonatal blood volume is equal or smaller than the pump prime which results in excessive dilution of all blood components. Blood is usually added to the he is priming volume to achieve hematocrit 21-24\%.during CPB. During CPB, mechanical ventilation is discontinued with maintenance of anesthesia and muscle relaxation. The degree of induced hypothermia during CPB is variable and depends on complexity of surgery, type of cardiac lesion and the surgeon preference [25]. Induced hypothermia may be mild, moderate, severe and deep hypothermic circulatory arrest. Perfusion flow and pressure should maintain MAP between 30 to $50 \mathrm{mmHg}$.

Ultrafiltration is a common technique, used in $99 \%$ of pediatric cardiac centers in North America [26]. Ultrafiltration removes excess fluid from the CPB circuit, increase hematocrit, remove inflammatory mediators and increase the concentration of platelets and plasma. After the repair of cardiac lesion, the patient can be separated from CPB after meeting the weaning criteria (resuming mechanical ventilation, normothermia, starting vasoactive drug infusion, acceptable heart rate and rhythm and normal arterial blood gases and electrolytes).

\section{Surgery without CPB}

Some forms of neonatal cardiac surgery are performed without the need for CPB and these include pulmonary artery banding, repair of coarctation of the aorta, and shunt procedures (modified Blalock-Taussig shunt or central shunt). The goal of pulmonary artery banding is to avoid excessive pulmonary blood flow until the neonate become older minimizing the risk of full correction of cardiac lesion, while that of shunt procedures is to increase pulmonary blood flow.

\section{Postoperative Management in NICU}

At the end of surgery all patients are transferred to NICU and kept on mechanical ventilator until stabilization of the patient status. The patient is fully monitored, slowly rewarmed to $37^{\circ} \mathrm{C}$ and vasoactive drug infusion is gradually decreased. All NICU personnel's should use PPE and avoid the contact with patient secretion to prevent transmission of SARS-CoV-2 infection.

\section{Conclusion}

There is no evidence of vertical transmission of SARS-CoV-2 infection from mother to her fetus but neonates born to infected mothers may catch infection during breast feeding. The goals of perioperative management for this group of babies who are submitted to urgent cardiac surgery include the use of PPE, preoperative stabilization of the neonatal hemodynamic status and the use of the smallest CPB priming volume.

\section{Acknowledgement}

None.

\section{Conflict of Interest}

No conflict of interest.

\section{References}

1. Zhu N, Zhang D, Wang W, Li X, Yang B, et al. (2020) A novel coronavirus from patients with pneumonia in China, 2019. N Engl J Med 382(8): 727733.

2. Wang C, Horby PW, Hayden FG, Gao GF (2020) A novel coronavirus outbreak of global health concern. Lancet 395(10223): 470-473.

3. Chen R, Chen J, Meng Q (2020) Chest computed tomography images of early coronavirus disease (COVID-19). Can J Anesth

4. Lu Q, Shi Y (2020) Coronavirus disease (COVID-19) and neonate: What neonatologist need to know. J Med Virol 1-4.

5. Chen H, Guo J, Wang C, Luo F, Yu X, et al. (2020) Clinical characteristics and intrauterine vertical transmission potential of COVID-19 infection in nine pregnant women: a retrospective review of medical records. Lancet 395(10226): 809-815.

6. Hoffman JI and Kaplan S (2002) The incidence of congenital heart disease. J Am Coll Cardiol 39(12): 1890-1900.

7. Pruetz JD, Carroll C, Trento LU, Chang RK, Detterich J, et al. (2014) Outcomes of critical congenital heart disease requiring emergent neonatal cardiac intervention. Prenat Diagn 34(12): 1127-1132.

8. Tasnim Agency (2020) Birth of a neonate from infected mother COVID-19 in Babol city.

9. Zarchia MZ, Neamatzadehc H, Dastgheibe SA, Abbasif H, Mirjalilid SR, et al. (2020) Vertical Transmission of Coronavirus Disease 19 (COVID-19) from Infected Pregnant Mothers to Neonates: A Review. Fetal Pediatr Pathol

10. Zeng H, Xu C, Fan J, Tang Y, Deng Q, et al. (2020) Antibodies in Infants Born to Mothers With COVID- 19 Pneumonia. JAMA

11. Dong L, Tian J, He S, Zhu C, Wang J, et al.(2020) Possible Vertical Transmission of SARS- CoV- 2 From an Infected Mother to Her Newborn. JAMA

12. Kimberlin DW, Stagno S (2020) Can SARS- CoV- 2 Infection Be Acquired in Utero? More Definitive Evidence Is Needed. JAMA

13. Zhu H, Wang L, Fang C, Peng S, Zhang L, et al. (2020) Clinical analysis of 10 neonates born to mothers with 2019-nCoV pneumonia. Transl Pediatr 9(1): 51-60.

14. Rasmussen SA, Kissin DM, Yeung LF, Mac Farlane K, Chu SY, et al. (2011) Preparing for influenza after 2009 H1N1: Special considerations for pregnant women and newborns. Am J Obstet Gynecol 204 (6 Suppl 1): S13-S20.

15. Watson T, Kakar P, Srivastava S, Dhanjal TS (2007) Eustachian valve remnant. Cardiol J 14(5): 508-509.

16. Delphin E, Seckin AI, Moore RA (2005) Preoperative evaluation and preparation of the pediatric patient with cardiac disease. In: Lake CL (edr), Pediatric Cardiac Anesthesia, $4^{\text {th }}$ (edn), Appleton \& Lange, USA, pp. 96-110.

17. Hensley F (1990) Premedication for children with congenital heart disease-beneficial or harmful? J Cardiothorac Anesth 4(4): 423-424.

18. Nabil A Mageed, Ibrahim I Abd El Baser, Hani I Taman (2020) Anesthetic management of surgical repair of coarctation of aorta in neonates and infants. Anaest \& Sur Open Access J 1(5): 2020

19. Nabil A Mageed, Ibrahim I Abd El Baser, Hani I Taman (2020) Perioperative Management of Pulmonary Hypertension in Pediatric Cardiac Surgery. Anaest \& Sur Open Access J 1(3). 
20. Zhao S, Ling K, Yan H, Zhong L, Peng X, et al. (2020) Anesthetic Management of Patients with COVID 19 Infections during Emergency Procedures. J Cardiothorac Vasc Anesth 34(5): 1125-1131.

21. Oklu E, Bulutcu FS, Yalcin Y, Ozbek U, Cakali E, et al. (2003) Which anesthetic agent alters the hemodynamic status during pediatric catheterization? Comparison of propofol versus ketamine. J Cardiothorac Vasc Anesth 17(6): 686-690.

22. Williams GD, Jones TK, Hanson KA, Morray JP, et al. (1999) The hemodynamic effects of propofol in children with congenital heart disease. Anesth Analg 89(6): 1411-1416.

23. Laird TH, Stayer SA, Rivenes SM, Lewin MB, Mc Kenzie ED, et al (2002) Pulmonary-to-systemic blood flow ratio effects of sevoflurane, isoflurane, halothane, and fentanyl/midazolam with 100\% oxygen in children with congenital heart disease. Anesth Analg 95(5): 1200-1206.

24. Bojan M(2019) Recent achievements and future developments in neonatal cardiopulmonary bypass. Paediatr Anaesth 29(5): 414-425.

25. Kotani Y, Tweddell J, Gruber P, Pizarro C, Austin EH, et al. (2013) Current cardioplegia practice inpediatric cardiac surgery: A North American multiinstitutional survey. Ann Thorac Surg 96(3): 923-929.

26. Harvey B, Shann KG, Fitzgerald D, Mejak B, Likosky DS, et al. (2012) International pediatric per-fusion practice: 2011 survey results. J Extra Corpor Technol 44(4): 186-193. 